\title{
On the prognostic potential of the sequential 123-I-HDA-tomoscintigram after the first MI
}

\author{
H. Rösler*, U. NoelpP*, T. Toth $\dagger$, P. A. Schubiger $\ddagger$ and H. R. Hunziker $\dagger$ \\ *Inselspital/Universität Bern, Nuclear Medicine Department, "Regionalspital Thun, Med. Klinik, \\ and $\ddagger$ Eidgenössisches Inst. $f$. Reaktorforschung Würenlingen, Switzerland
}

- KEY WORDS: 123-I-heptadecanoic acid, myocardial tomoscintigraphy, first-pass radioangiocardiography, myocardial ischaemia, myocardial infarct.

\section{Introduction}

Myocardial tomoscintigraphy using 123-I-heptadecanoic acid (HDA) is highly sensitive in confirming, localizing and sizing acute myocardial infarcts. A circumscribed decrease in HDA uptake immediately

- after i.v. administration is the clear-out criterion $^{(1-3)}$.

The regional elimination of HDA, visualized by a second tomoscan $30 \mathrm{~min}$ after injection, was found to be homogeneous over a healthy myocardium. The elimination is delayed in some myocardial infarcts (MIs) with low HDA uptake (=cool lesion). These lesions show a cool-warm behaviour (=d-type lesion) and were more often seen in the early stages of infarction than in scars which follow a cool-cool (=c-type) or cold-cold (=e-type lesion) sequence. Akinetic and hypokinetic zones in the first pass examination were found to be larger than the myocardial defect with c- and e-type and smaller with d-type lesions (2). The cool-warm behaviour seems to be indicative of viable myocardium with an abnormal HDA degradation, in contrast to cool-cool lesions in which necrosis and/or scarring predominate. Lesions with a 'delayed downslope' have been interpreted as being ischaernic ${ }^{(1,3-8)}$.

For myocardial lesions with residual uptake but prolonged HDA catabolism, one suspects that cellular disintegration is not complete, but that it may continue. Thus, the regional necrosis and, consequently, pump failure could increase over the following days and weeks. On the other hand, recovery from transient ischaemia should also be possible, leading to a smaller defect and continuously improving cardiac function.

In serial examinations with the first tomoscans within 10 days after $\mathrm{MI}$ and the second 5-6 months

\footnotetext{
Address for correspondence Prof. H. Rösler, MD, CH-3000 BernInselspilal, Swilzerland.
}

later, we followed patients with different initial HDA patterns. We intended to determine those criteria seen in the first HDA tomoscintigram which would have prognostic significance for the individual patient. Additional first-pass radiocardiography helped us to define the location and extent of wall motion abnormalities and also gave us a quantitative measurement of the left heart function with the ejection fraction (EF).

\section{Methods}

123-I-HDA was delivered once a week $(40 \mu \mathrm{g}$ HDA ml $\mathrm{ml}^{-1}, 2 \mathrm{mCi} \mathrm{ml}^{-1}$ in a solution of lecithinglycocholic acid mixed micelles ${ }^{(9,20)}$. After the $i . v$. administration of 3-4 mCi, the LFOV camera with the 7-pinhole collimator needed-after patient positioning during the first $5 \mathrm{~min}$-another $12 \mathrm{~min}$ for the first, i.e. 'early', image. The patient remained on the table and the detector position was unchanged. At $30 \mathrm{~min}$ after the injection, a second image $(2 \times 500$ Kcounts $)$ was recorded by the computer over $18 \mathrm{~min}$. Three slices (apical, middle and basal) were reconstructed using the original Vogel-Kirch program. These printouts contained the data aquired early and late, each with different symbols. After a step-by-step calculation over all 60 radii, the retention rates (count density [late]/count density [early]) were drawn as an additional line which, in normals, is a rather straight one at a level between 0.61 and $0.98^{(2)}$. The size of a lesion was defined as the distance between the two crossing points of the individual (early or late) curve with the reference line for normals, in degrees or in $\%$ of $360^{\circ}$. For this study, however, the size was calculated as the average value over the three slices (Figs $I$ and 2). For a d-type lesion the retention rate was postulated to be more than $+30 \%$ (based on the minimal value found over the normal myocardium). 


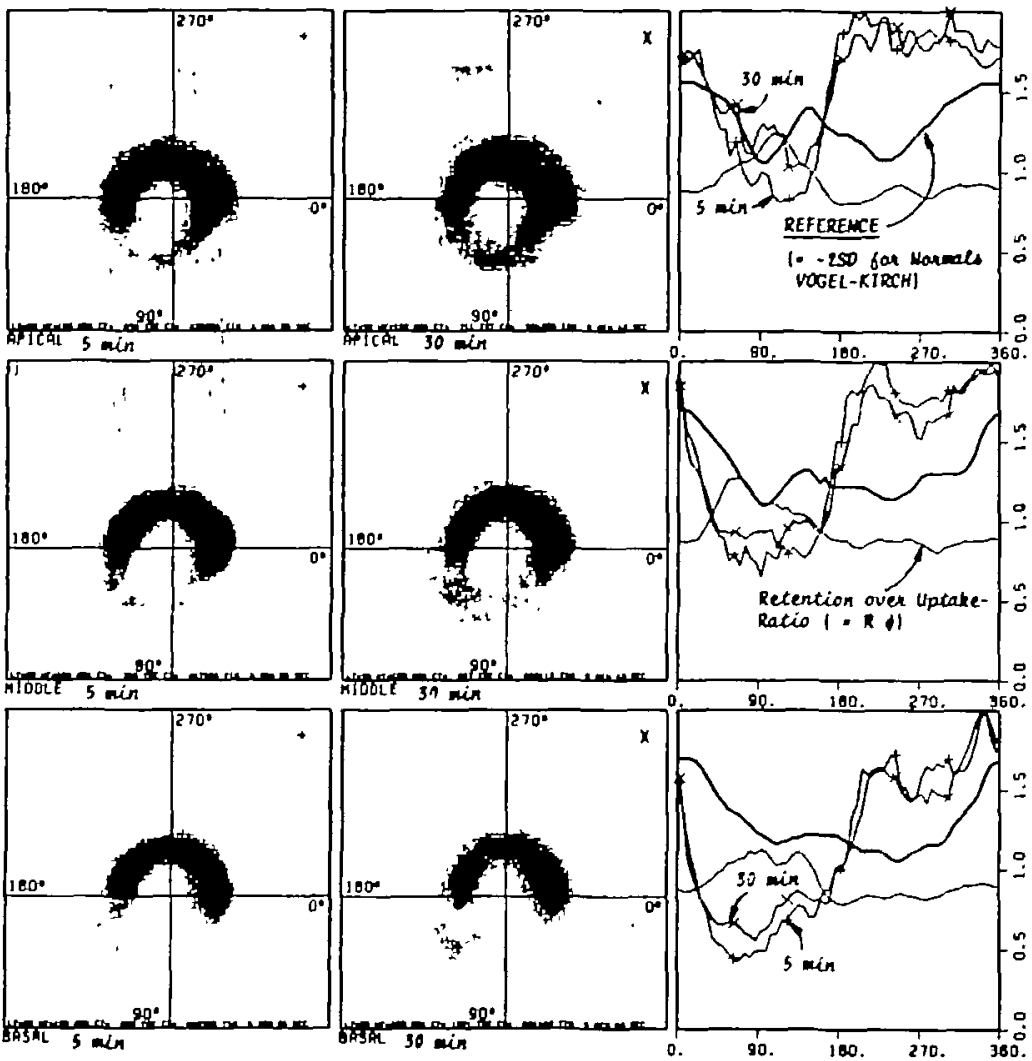

Figure 1(a)

Thus, the size of a d-type lesion, i.e. those parts of the defect and its borders with cool-warm behaviour, could be quantified and given again in degrees or $\%$ of $360^{\circ}$.

After changing the collimator (planar, low energy, high sensitivity) a first pass was acquired in $30^{\circ} \mathrm{RAO}$ projection (20 frames $\mathrm{s}^{-1}$ ) after a bolus injection of $20 \mathrm{mCi} 99 \mathrm{~m}$-Tc-albumine. Using a semiquantitative contour finding algorithm, the heart was imaged in enddiastolic (EDV) and endsystolic volume (ESV), followed by an EF calculation. Maps of wall motion, regional EF and diastolic as well as systolic positive and negative trends ${ }^{(11,12)}$ : (interobserver variance for EF calculations $r=0.99$ ) were added. As an equivalent for ED and ES volumes, the areas were quantified with a planimeter and given in arbitrary units. Statistics were based upon $t$-test calculations for pairs.

\section{Patients}

We started our prospective study with 98 patients but stopped after 35 patients had been recalled for the second examination. Of these, 4 had since died; 5 examinations could not be used because of technical problems (one or more computer data sets/patient were not evaluable). All patients had the first examination within the first 10 days, the second $19 \cdot 7 \pm 4.3$ weeks (i.e. 5 months) after their MI.

\section{Results}

Figure 1 shows the original plots of a patient with a posterior to postero-septal MI 9 days old [Fig. 1(a)]. The size of the defect is defined by the distance between both of the crossing points of the circumferential profile count measurement (given in lines with + symbols for an early image) and the reference line. This defect seems to be less pronounced in the late (i.e. $30 \mathrm{~min}$ ) scintigraphic image as well as in the extent of the second profile counts (with $\times$ symbols). The scintigraphic pattern did not change dramatically with time, but the ischaemic score, which was high initially (=steep increase of the retention-over-uptake ratio), showed 


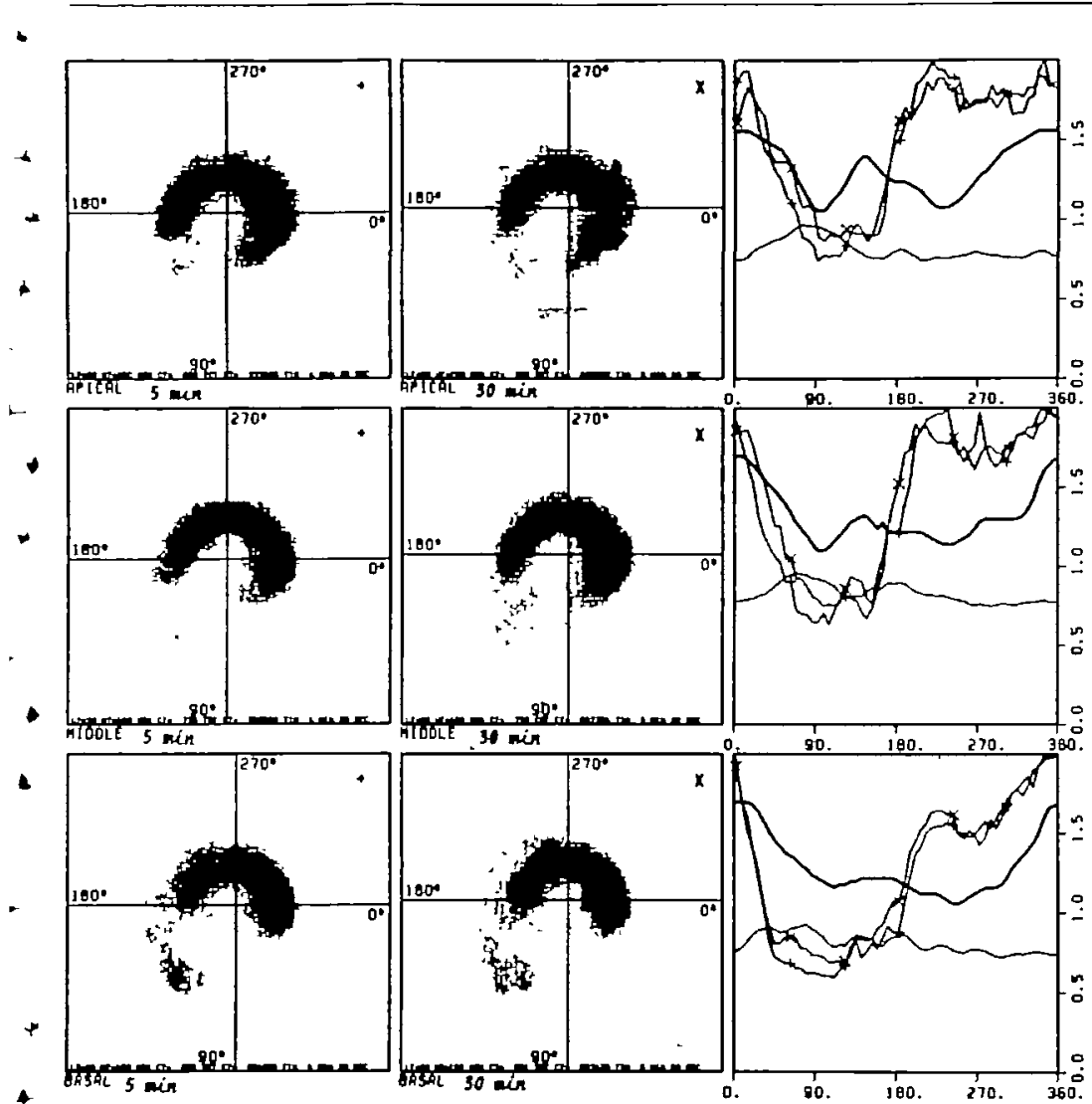

Figure I(b) Two sets of three tomoshces each and their quantitative evaluation (see text). A nine-day old postero-septal MI with a high d-type score [Figure $/(a)$ ] is compared with a second HDA tomoscan done 5 months later, when a cool-cool behaviour dominates the lesion of non-altered size and identical localization.

a marked reduction (this curve is flattened in Fig. l(b) but not normalized).

In Fig. 2, a patient suffering from an anterior MI, there is a slight reduction in the size of the defect (demonstrated with selected middle planes only), the second examination being done 16 weeks later. Was

the extent of the shrinking of this lesion predictable with the initial high ischaemia score?, and why did

- this lesion only decrease and not completely disappear? Both figures demonstrate the reproducibility of individual defects and the consistency of a finding over time.

The initial size of an HDA-uptake defect (=HDA defect), as given in degrees, leads to a corresponding decrease in the pump function of the left ventricle, as measured with the left heart-ejection fraction (= LH-EF in Table 1). As was to be expected, the correlation was found to be linear and inverse, with a mean EF value of $64 \%$ with no defect (extrapolated) and an $E F=38 \%$ for a $200^{\circ}$ defect.
With an increasing defect size, the left ventricle tends to dilate (Table 1, 2-5).

In general, the MI defects showed a tendency to shrink with time; the $\triangle \mathrm{HDA}$ defect, calculated as the percental decrease from the first to the second examination, was $-13.56( \pm 32.3) \%$. This decrease was correlated with an increasing EF, and with decreasing left ventricular volume (Table 1, 6-8). These results confirm both examinations as being useful in the follow-up of MI patients.

There is a coarse correlation between the size of an ischaemic region (=d-type lesion) and the total HDA uptake defect size (Table 1, 9). But, this initial cool-warm behaviour itself is by no means a predictor for the later outcome: the EF, does not increase with higher d-type scores, neither does the end-systolic volume shrink. Only a slightly better reduction of the end-diastolic volume occurs with an initially more extensive ischaemic lesion (Table 1, 10-14). 


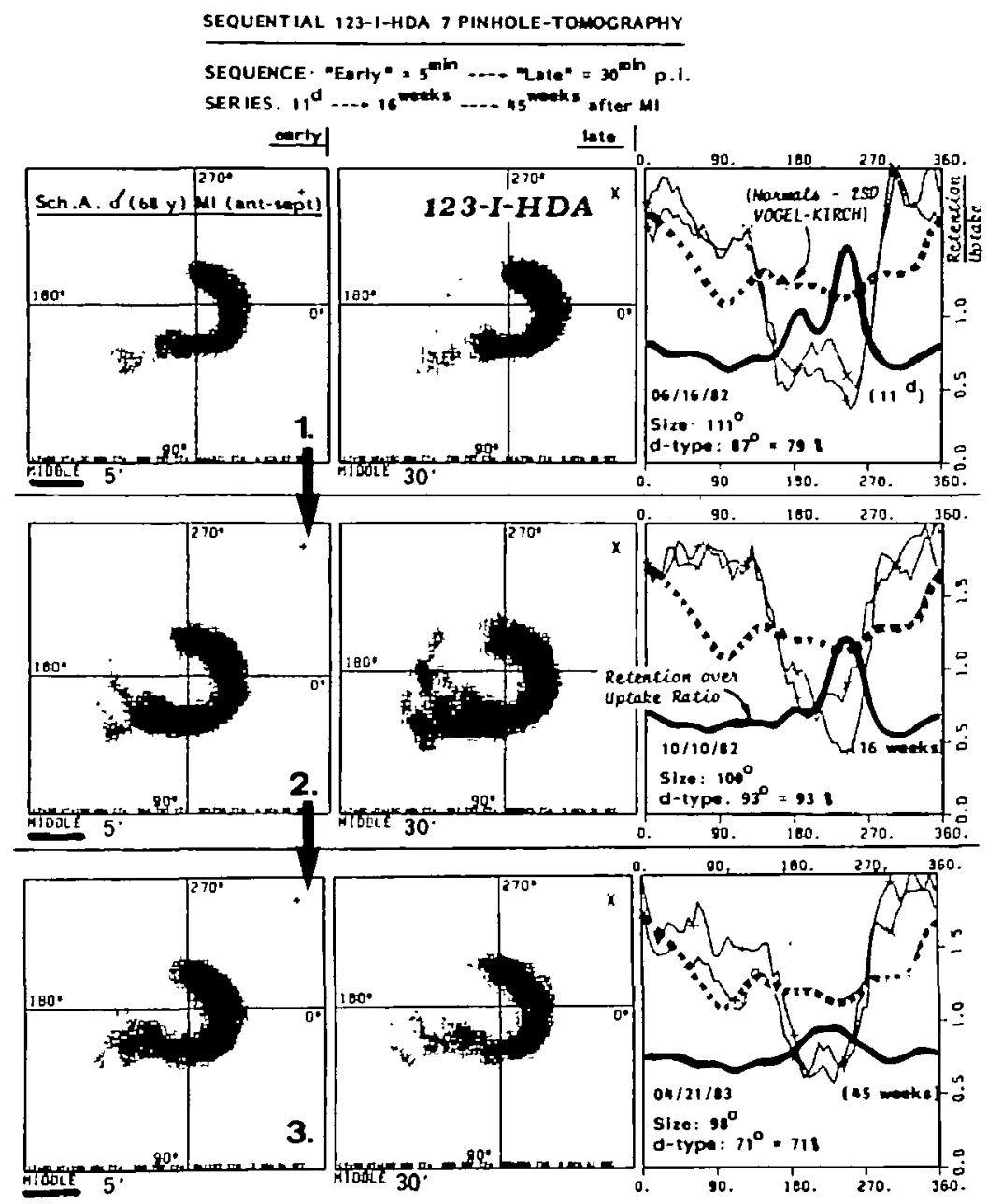

Figure 2 A sequence of three tomoscans (shown are the middle planes only) from the 11 th day over 16 weeks to 45 weeks after a mainly anterior infarction. There is only a small reduction of the lesions's size, in spite of a high d-type score with the initial examination.

Comparing those MIs with a high ischaemic score (i.e. with a d-type behaviour over more than $50 \%$ of the lesion) with mainly c-type lesions, one finds a trend. But the difference between initially high ischaemic lesions which greatly decrease and the lesser ischaemic ones which shrink less, cannot be proved statistically (Table 2 ).

We also suspected a different behaviour between initially large and small MIs. But again, there are no strong correlations (Table 3): trends for a greater decrease of highly ischaemic lesions in both groups cannot be ascertained.

To prove these disappointing results, the initial findings were revised retrospectively, taking into consideration the later outcome: the alteration of a MI-uptake defect over time is neither a function of its initial size nor of the initial d-type extension (Table 4).

\section{Discussion}

\section{THE METHOD}

For dynamic tomography, simple detection of a lesion is not enough: one needs to know its exact location, size, shape, and integration into the functional structure of the organ itself ${ }^{13}$.

These demands may be fulfilled by 7-pinhole tomography. Because of its modest tomographic 
Table I Correlations

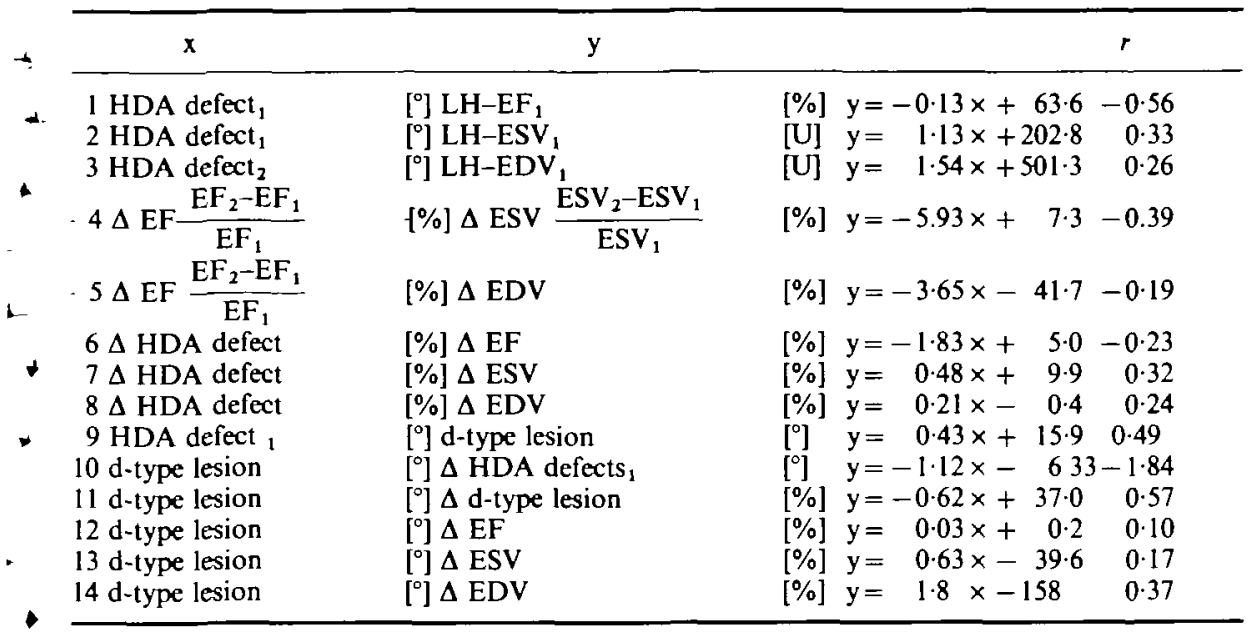

ability, its substantial interplanar propagation, and-most importantly-the difficulties inherent in its correct positioning, the advantages of 7-pinhole tomography are often forgotten. These are fair counting efficiency and considerable contrast enhancement, as well as quantitative possibilities in

Table 2 Alteratton of a MI size over $\sim 5$ months, depending on the initial d-type score

\begin{tabular}{|c|c|c|c|}
\hline \multirow[b]{2}{*}{$N$} & \multicolumn{2}{|c|}{ Amount of d-type: } & \multirow{2}{*}{$\begin{array}{c}\text { Decreasing after } \sim 5 \text { months } \\
(\Delta \text {-size in } \% \text { initial MI })\end{array}$} \\
\hline & $>50 \%$ & $<50 \%$ & \\
\hline $\begin{array}{l}13 \\
14\end{array}$ & $91 \cdot 3 \pm 31 \cdot 7^{\circ}$ & $24 \cdot 0 \pm 15.9^{\circ}$ & $\left.\begin{array}{r}-17.5 \pm 33.1 \% \\
-9.9 \pm 26.0 \%\end{array}\right\} P>0.5$ \\
\hline
\end{tabular}

$\Delta$ size of a MI-uptake defect $=$ decrease in both MIs: those with a high and those with a low 'ischaemia score'. The difference between the two groups is not significant. describing regional activity densities, in determining the size of lesions and in describing any abnormalities. These distinctive features made this technique indispensable for us. Even today, there are no techniques available for continuous data collection, simultaneously from all left ventricular segments; no other method, which allows one to find all lesions and to measure all regions equally and fast. There is one handicap, just as with planar techniques: absolute uptake values cannot be ascertained. Theoretically, any time course, i.e. a velocity constant, could be derived from many separate points of the left heart myocardium; but, in order to include the first minutes after application, one needs an additional and troublesome primary positioning of the detecting device and some kind of bloodpool background subtraction.

It has been postulated, that iodinated tracers do

Table 3 Comparison of large and small MIs: both show a shrinkage which is independent of the intial d-iype score

Follow-up $\sim 5$ months [ $\%$ initial value]

\begin{tabular}{|c|c|c|c|c|c|}
\hline$N$ & Initial $\mathrm{MI}$ size $\left[{ }^{\circ}\right]$ & Inutial d-type [\%] & $\Delta$-size in $\%$ initi & ial $\mathrm{MI}$ & $\Delta E F$ \\
\hline \multicolumn{6}{|c|}{ Large $M I$ defects } \\
\hline $\begin{array}{l}9 \\
6\end{array}$ & $\left.\begin{array}{l}133.3 \pm 27.6 \\
165.0 \pm 38.4\end{array}\right\} N S$ & $\left.\begin{array}{l}87.6 \pm 20.8 \\
29 \cdot 2 \pm 15.5\end{array}\right\} P<0.0001$ & $\left.\begin{array}{l}-16 \cdot 1 \pm 31 \cdot 1 \\
-10 \cdot 7 \pm 37 \cdot 1\end{array}\right\}$ & NS & $\left.\begin{array}{r}3 \cdot 0 \pm 10 \cdot 2 \\
-2 \cdot 3 \pm 14 \cdot 3\end{array}\right\} \mathrm{NS}$ \\
\hline \multicolumn{6}{|c|}{ Small MI-defects } \\
\hline $\begin{array}{l}4 \\
8\end{array}$ & $\left.\begin{array}{l}71 \cdot 3 \pm 19 \cdot 2 \\
52 \cdot 8 \pm 20-1\end{array}\right\} \mathrm{NS}$ & $\left.\begin{array}{l}99.8 \pm 52.3 \\
20.1 \pm 16.2\end{array}\right\} P<0.0001$ & $\left.\begin{array}{r}-21 \cdot 0 \pm 42 \cdot 4 \\
-7.8 \pm 17.5\end{array}\right\}$ & NS & $\left.\begin{array}{r}8.8 \pm 15.9 \\
-1.5 \pm 7.7\end{array}\right\} N S$ \\
\hline
\end{tabular}

In both large and small initial MI-uptake defects, the defect size decreases over $\sim 5$ months and EF increases slightly-differences are not significant. Within both groups these results are independent of the initial 'ischaemia score'. 
Table 4 The alteration of the MI size is not dependant on the initial d-type-score; an evaluation in retrospect

d-Type behaviour

$\Delta$ MI-size over 5 months Initial MI-size (uptake defect)*

in $\%$ of the MI-sizet

\begin{tabular}{|c|c|c|c|c|c|c|}
\hline $\begin{array}{l}\text { (a) Decreasing }>20 \% \\
\text { (b) Decreasing }<20 \%,>0 \% \\
\text { (c) Increasing }\end{array}$ & $\begin{array}{l}(-48 \cdot 8 \%) \\
(-7.1 \%) \\
(+12 \cdot 8 \%)\end{array}$ & $\begin{array}{r}118 \cdot 8^{\circ} \\
115 \cdot 8^{\circ} \\
84 \cdot 1^{\circ}\end{array}$ & $P>0.5$ & $P<0.5>0.2$ & $\left.\begin{array}{l}50.3 \% \\
68.6 \% \\
45.8 \%\end{array}\right\} P>0.5$ & $P>0.2<0$ \\
\hline
\end{tabular}

* $\Delta$-size of a MI-uptake defect is not a function of the initial size.

$+\Delta$-size of a MI-uptake defect is not a function of the initial d-type extension.

not provide a direct assessment of metabolic function as do 'natural', i.e. 11-C or 15-0 tracers. However, they provide an index of comparison, and even if this index can be affected by nonspecific conditions ${ }^{(14)}$, one can depend on the results in a setting such as ours.

\section{THE RESULTS}

Myocardial lesions can be visualized with 123-IHDA tomography and, thus, establish or-at leastconfirm the diagnosis. Moreover, this examination adds information as to size, shape and localization of an MI, all of which are of prognostic relevance $^{(13)}$. It is not by any means the only modern technique capable of doing this. In looking for an exclusive advantage in the use of HDA, we and other groups hoped to find other criteria of prognostic significance. Hope was placed in HDA and its ability to differentiate myocardial lesions due to their functional patterns, which could then be correlated with the patients' later outcome.

Is the lesion ischaemic, necrotic, or a scar? To be able to answer this question would result in important prognostic information. Indeed: there are two distinct patterns seen in MIs: one with a cool-cool behaviour, the other with a cool-warm sequence. The former (c-type) seems to dominate with an irreversible, stabilized condition, the other (d-type) $^{(2)}$ is attributed to ischaemia, i.e. to viable myocardium which is not irreversibly injured ${ }^{(14)}$ and, thus, non-stable myocardium.

Our patients were examined after the first (i.e. 'ischaemic') and the second (i.e. 'necrotic') stages of myocardial infarction ${ }^{(15)}$, in which the magnitude of territorial necrosis was already established. Ventricular performance depends on the residual viable myocardium. With an increasing defect, ventricular dysfunction must also increase. The patient's risk would increase because of induction of arrhythmia or by further decrease in the EF. Does this residual myocardium, later on, find support from the myocardium being primarily ischaemic which improves the global haemodynamics?; or does the patient remain at risk, because a mainly ischaemic lesion is going to be converted finally into a necrosis, thus, increasing the infarct's size to a larger scar?

Most of our patients experienced a decreasing size of their myocardial lesions. C-type, i.e. cool-cool lesions, shrunk by less than $10 \%$ (Table 2); i.e., with progressive fibrotic invasion a smaller, more compact scar resulted. But in the initially predominantly ischaemic lesions, i.e. those of the d-type (Table 2: $90 \%$ of the MI region followed a cool-warm sequence), the defect was twice as much reduced in its size with the later examination. This can be best explained by progressive reconversion of ischaemic to normal myocardium. The contracting muscular mass and the EF thus increase, and risks from the jeopardized conduction system are lessened. These results represent trends. From a statistical point of view, they are not without doubt. Therefore one should be careful in assigning these findings directly to an individual patient. One should also be aware that we cannot always expect clear-cut c- or d-type lesions.

At least the cool-cool and cold-cold infarcts are definitely necrotic. When examined more than one week after MI, these lesions are stabilized: this means that for most of the patients the endpoint of progressive left heart failure after MI has been reached.

D-type lesions must be interpreted with much more caution. Theoretically, considerable progress in regaining pump function is predictable, but this cannot be proven statistically. What happens to the individual is not foresceable, at least not after only one early examination since 'only ischaemia' from one end of the lesion to the other, as well as varying mixtures of ischaemic and necrotic tissues within such a lesion, may be the substrate for such a cool-warm lesion. We are not able to identify these subtypes, nor are we able to forecast their different futures. 
Our results have encouraged us to follow-up more patients with serial 123-I-HDA tomoscintigraphies. Handicaps of the introductory period-irregular delivery of the substance and difficulties with the

* tomotechniques-have been overcome one must look for a simpler way to evaluate the findings - output from the computer.

\section{Summary}

Seven-pinhole tomography (SPT) using 123-Iheptadecanoic acid (HDA) is highly sensitive in confirming, sizing and localizing myocardial infarcts (MIs). Its prognostic value, however, is unknown. - Thirty-five consecutive patients coming for a routine SPT within 10 days after MI underwent a second - examination $\sim 20$ weeks later. These examinations combined an early $(5 \mathrm{~min})$ and a late $(30 \mathrm{~min}) \mathrm{SPT}$ after administration of $4 \mathrm{mCi}$ 123-I-HDA, as well as a first pass radiocardioangiography. Scars and infarcts without residual HDA catabolism were related to lesions which followed (from $5 \mathrm{~min}$ to $+30 \mathrm{~min}$ ) a cool-cool or cold-cold behaviour. Cool-warm lesions (due to low HDA uptake and

- prolonged washout) were considered ischaemic. This ischaemic behaviour could not be correlated to the later course of the illness: in those patients with higher initial ischaemic scores, the ejection fraction did not increase disproportionately nor was the size $\rightarrow$ of the lesion significantly smaller in the later examination than in those patients with lower ischaemic scores. Only a slightly better reduction of the end-diastolic volume was found in the patients with initially predominantly ischaemic lesions. Thus, SPT with HDA seems to be of limited value for an early prognostic evaluation of patients with $\mathrm{MI}$.

We wish to thank Nancy Studer-Pantaleo for the preparation of the manuscript of this paper.

\section{References}

(1) Poe ND, Robinson GD, Zielinski FW et al. Myocardial imaging with 123-I-hexadecenoic acid. Radiology 1977; 124: 419-24.

(2) Rösler H, Hess T, Weiss M et al. Tomoscintigraphic assessment of myocardial metabolic heterogenity. J Nucl Med 1983; 24: 285-96.

(3) Van der Wall EE, Westera G, Den Hollander Wet al. External detection of regional myocardial metabolısm with radioiodinated hexadecanoic acid in the dog heart. Eur J Nucl Med I98I; 6: 147-51.

(4) Feinendegen LE, Vyska K, Freundlieb $\mathrm{CH}$ et al Noninvasive analysis of metabolic reactions in body tissue, the case of myocardial fatty acids, Eur J Nucl Med 1981; 6: 191-200.

(5) Huckel VF, Lyster DM, Morrison RT. The Potential role of 123-iodine-hexadecanoic acid in assessing normal and abnormal myocardial metabolism. J Nucl Med 1980; 21: p 57.

(6) Lerch RA, Ambos HD, Bergmann STR, welch MJ, Ter-Pogossian MM, Sobel BE. Localization of viabel, ischaemic myocardium by positron-emission tomography with 11-C-palmitate. Circulation 1981; 64: 689-99.

(7) Lerch RA, Bergmann STR, Ambos HD, Welch MJ, Ter-Pogossian MM, Sobel BE. Effect of flowindependent reduction of metabolism on regional myocardial clearance of 11-C-Palmitate. Circulation 1982; 65: 731-8.

(8) Van der Wall EE, Heidendahl GAK, Den Hollander W. Metabolic myocardial imaging with 123-I-labelled heptadecanoic acid in patients with angina pectoris. Eur J Nucl Med 1981; 6: 391-6.

(9) Argentini M, Zahner M, Schubiger PA. Comparison of several methods for the synthesis of $\omega$-iodine-123heptadecanoic acid. J Radioanal Chem 1981; 65: 131-8.

(10) Schubiger PA, Argentini M, Blāuenstein P, Lerch R. Mixed micells: A new problem-free solution for $\omega$-I 123-heptadecanoic acid in comparison. Nuklearmedizin 1984; 23: 27-8.

(11) Fritschy P. Ein halbautomatisches Programm für die Auswertung von Radioventrikulogrammen in der Erstpassagetechnik. Fortschr Rōntgenstr 1981; 134: $386-92$

(12) Noelpp U, Schad N, Rösler H. Trendszintigraphie. Nucl Med 1977; 16: 232-7.

(13) Keyes JW Jr. Perspectives on tomography. J Nucl Med 1982; 23: 633-40.

(14) Goldstein RA. Myocardial metabolic imaging: A new diagnostic era. J Nucl Med 1982; 23: 641-4.

(15) Swan HJC, Shah PK, Rubin ST. Role of vasodilators in the changing phases of acute myocardial infarction. Am Heart J 1982; 103: 707-715. 